Revisión

\title{
Riesgo psicosocial de los trabajadores inmigrantes en España
}

\section{Psichosocial risk of inmigrant workers in Spain}

\author{
Sanchis-Ruiz, A. ${ }^{\text {; }}$ Varela-Hall, C. ${ }^{2}$; Martins-Muñoz, G. ${ }^{3}$, y Díaz-Sotero, M. ${ }^{4}$ \\ 1. Residente Medicina del Trabajo Hospital Infanta Leonor, Madrid. España. \\ 2. Residente Medicina del Trabajo Fundación Jiménez Díaz, Madrid. España. \\ 3. Residente Medicina del Trabajo Hospital Clínico San Carlos, Madrid. España. \\ 4. Residente Medicina del Trabajo Hospital Clínico San Carlos, Madrid. España.
}

Recibido: 13-05-13

Aceptado: 23-07-13

\section{Correspondencia}

Antonio Sanchis Ruiz

Residente Medicina del Trabajo

Servicio de Prevención de Riesgos Laborales

Hospital Universitario Infanta Leonor

Avenida Gran Vía del Este, 80

28031 Madrid (España)

Teléfono: 911918013

Correo electrónico: sarua1983@gmail.com

Resumen

Introducción: España en las últimas décadas ha sido un receptor de un intenso flujo migratorio, sin embargo, por la crisis financiera actual, se está evidenciando un gran descenso. La inmigración está ligada al trabajo y búsqueda de mejores condiciones de vida. El trabajador inmigrante presenta una serie de riesgos psicosociales.

Metodología: Se realiza búsqueda en principales bases de datos bibliográficas mediante palabras clave y se descriptores, aplicando posteriormente criterios de pertinencia, inclusión y exclusión, seleccionándose un total de 15 artículos que se clasifican mediante los criterios de evidencia del Scottish Intercollegiate Guidelines Network (SIGN).

Resultados: Ser inmigrante conlleva a mayor discriminación, racismo y xenofobia en el lugar del trabajo. Las mujeres son más vulnerables a riesgos psicosociales. La mayoría de estudios resaltó la existencia de precariedad laboral. También hay discriminación más notable con ciertas nacionalidades. Muchos inmigrantes con estudios de alto nivel, se dedican a trabajos poco cualificados.

Discusión: La mayor parte de los artículos revisados consideran que las condiciones de trabajo del inmigrante en España están caracterizadas por la precariedad, inestabilidad y por tanto vulnerabilidad en el ámbito laboral. También hay peor salud auto percibida, distrés físico y mayor riesgo de trastornos mentales. Diferencias de incidencia de acuerdo al género, siendo el sexo femenino más vulnerable.

Conclusiones: En los artículos revisados la población inmigrante, en su mayoría, procede de países de menores rentas. Es relevante la discriminación percibida por un alto porcentaje de los inmigrantes en España en el entorno laboral. En general, el inmigrante se ve limitado en cuanto al tipo de trabajo al que accede, sus condiciones y derechos laborales.

Med Segur Trab (Internet) 2013; 59 (232) 345-360

Palabras clave: Trabajador inmigrante, riesgo psicosocial, España. 


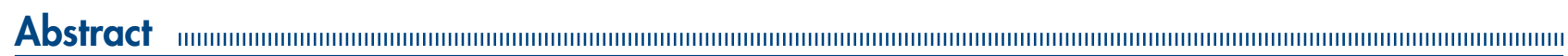

Introduction: During the last decades, Spain has been a receptor of an intense migratory flow, however, by the current financial crisis, it is showing a great fall. Immigration is linked to work and the search of better living conditions. The immigrant worker has several psychosocial risks.

Metbods: We performed a search in the main bibliographic databases using key words and descriptors, subsequently applying relevance, inclusion and exclusion criteria, selecting a total of 15 articles to which we applied the evidence criteria of the Scottish Intercollegiate Guidelines Network (SIGN).

Results: Being an immigrant leads to greater discrimination, racism and xenophobia at the workplace. Women are most vulnerable to psychosocial risks. Most of the studies also highlighted the existence of job insecurity. There is more discrimination among some nationalities. Many immigrants with a high level of studies, dedicate to low qualified jobs.

Discussion: Most of the studies reviewed considered that work conditions in the immigrant in Spain are characterized by precariousness, unsteadiness and therefore vulnerability in the occupational sphere. There are also poor self-perceived health, physical distress and major risk of mental disorders. Incidences differ on gender, where females are more vulnerable.

Conclusions: In the articles reviewed, immigrant population mostly comes from countries with lower incomes. It is outstanding the perceived discrimination on this field by a high percentage of the immigrants in Spain. Generally, the immigrant is limited in type of work that accesses, conditions and rights.

Med Segur Trab (Internet) 2013; 59 (232) 345-360

Key Words: Inmigrant worker, psicosocial risk, Spain. 


\section{INTRODUCCIÓN}

España ha pasado de ser un país tradicionalmente exportador de emigrantes a ser uno receptor de un intenso flujo migratorio de personas que proceden principalmente de países de baja renta. Según datos del Ministerio de Empleo y Seguridad Social el número de extranjeros con certificado de registro o tarjeta de residencia en vigor a 30 de septiembre de 2012 es superior a los 5 millones $^{1}$. Sin embargo, según la Organización para la Cooperación y el Desarrollo Económico (OCDE) ${ }^{2}$, los flujos anuales de inmigración permanente disminuyeron drásticamente: de casi 700 mil en 2007 a prácticamente 300 mil en 2010.

Las corrientes globales de inmigración en España parece que continuaron disminuyendo en 2011, aunque en menor medida. La inmigración es un fenómeno íntimamente ligado al trabajo, y la búsqueda de mejores condiciones de vida es una de las principales causas que justifica la migración. Según Martínez M., la inmigración es un fenómeno social emergente con notable impacto en el ámbito sanitario. Aunque el proceso migratorio puede darse por diversas causas, la principal es la integración en el mercado laboral, lo que supone vencer determinadas barreras el proceso de adaptación cultural en el país de destino, entre ellas y como principales, las idiomáticas y las sociales ${ }^{3}$.

Existe una tendencia a que la población inmigrante trabaje principalmente en los sectores de servicios y construcción, seguido de industria y agricultura, tomando especial peso el sector de servicios domésticos y hostelería. En común para todos estos sectores de principal dedicación inmigrante es que se caracterizan por peores condiciones de empleo, mayor temporalidad, precariedad, discriminación y dificultad para acceder a otros puestos de trabajo de mayor cualificación o de promoción. Este hecho, que afecta a inmigrantes con independencia de su situación legal, conlleva una serie de efectos psicosociales perjudiciales para su salud, afectando el eje físico, social, psicológico y cultural. Según Gálvez Herrer, los procesos migratorios no suponen per se un problema de salud mental, pero sí plantean situaciones de alta vulnerabilidad y retos de integración ${ }^{4}$.

El nivel de estrés por exposición a riesgos psicosociales aumenta en la sociedad actual, en la que hay mayores exigencias laborales, requiriendo mayor amplitud de destrezas y funciones, mayor desequilibrio trabajo-familia, incrementándose a su vez los trabajos temporales, la subcontratación y la falta de estabilidad en el empleo ${ }^{5}$.

Diversos trabajos que han estudiado la salud de los inmigrantes ponen de manifiesto que se trata de una población joven y sana, que utilizan en menor medida los servicios sanitarios en los primeros años de su residencia en el país al que migran ${ }^{6,7}$. Son varias las razones por las que esto ocurre, ya sea por situación irregular, por desconocimiento del funcionamiento del sistema, por poca conciencia de enfermedad, o por malas experiencias con otros sistemas sanitarios, entre otras ${ }^{8}$.

Son escasos los datos conocidos en relación a los riesgos psicosociales de los trabajadores inmigrantes y las herramientas con las que se deben controlar o eliminar dichos riesgos. Este trabajo pretende revisar la literatura publicada durante los últimos 10 años, sobre riesgos psicosociales en el trabajador inmigrante en España, al objeto de identificar los principales riesgos psicosociales en esta población, conocer sus principales características epidemiológicas, su etiología y patrón de presentación, así como determinar las principales medidas de prevención de riesgo psicosocial en esta población.

\section{METODOLOGÍA}

Para realizar la revisión bibliográfica se ha buscado literatura científica en bases de datos nacionales e internacionales, especializadas y generales, incluyendo MEDLINEPubMed, EMBASE, Cochrane Public Health Group, PsycINFO, LILACS, SciELO, CISDOC 
así como en otras bases de datos científicas más locales como IME (Indice Médico Español, con revistas de ciencias de la salud), ISOC (revistas de ciencias sociales y humanidades), IBSS y TESEO (tesis doctorales presentadas en las universidades españolas desde 1976).

Para elaborar la estrategia de búsqueda se utilizaron los siguientes términos: inmigrante, trabajador inmigrante, salud mental, mental, psicosocial, riesgo psicosocial, España, trabajo y riesgo, que se encuentran detallados en la Tabla I.

Tabla I. Relación de términos de búsqueda utilizados en las diferentes bases de datos y el número de artículos seleccionados de cada una

\begin{tabular}{|c|c|c|c|c|}
\hline & \multicolumn{2}{|c|}{$\begin{array}{l}\text { Términos incluidos en la estrategia } \\
\text { de búsqueda }\end{array}$} & $\begin{array}{l}\text { N. }{ }^{\circ} \text { de artículos } \\
\text { resultado } \\
\text { de la búsqueda }\end{array}$ & $\begin{array}{c}\mathrm{N}^{\circ} \text { de artículos } \\
\text { seleccionados } \\
\text { por resumen (abstract) }\end{array}$ \\
\hline \multirow[t]{11}{*}{ PUBMED } & \multirow{4}{*}{$\begin{array}{l}\text { Immigrant workers } \\
(773)\end{array}$} & + Spain & 40 & 8 \\
\hline & & + psychosocial & 12 & 5 \\
\hline & & $\begin{array}{l}+ \text { psychosocial work } \\
\text { characteristics }\end{array}$ & 2 & 2 \\
\hline & & + psychosocial health & 59 & 10 \\
\hline & Migrant workers & + psychosocial health & 1 & 0 \\
\hline & $\begin{array}{l}\text { Emigrant and } \\
\text { immigrant }\end{array}$ & + work + psychosocial & 10 & 9 \\
\hline & \multirow[t]{2}{*}{ Immigrant workers } & $\begin{array}{l}+ \text { psychosocial health } \\
+ \text { Spain/spaniards }\end{array}$ & 121 & 16 \\
\hline & & + health & 88 & 11 \\
\hline & $\begin{array}{l}\text { Immigrant mental } \\
\text { health }\end{array}$ & + Spain & 45 & 27 \\
\hline & \multirow{2}{*}{$\begin{array}{l}\text { Immigrant work } \\
\text { Spain }\end{array}$} & + mental & 8 & 8 \\
\hline & & + psychosocial & 6 & 6 \\
\hline CISDOC & Immigrant workers & & 26 & 6 \\
\hline \multirow[t]{4}{*}{ OSH UpDate } & \multirow{4}{*}{$\begin{array}{l}\text { Immigrant workers } \\
(422)\end{array}$} & + Spain & 1 & 1 \\
\hline & & + mental health & 0 & \\
\hline & & + risk & 0 & \\
\hline & & + psychosocial & 0 & \\
\hline \multirow[t]{3}{*}{ COCHRANE } & \multirow[t]{2}{*}{ Immigrant } & + worker & 3 & 1 \\
\hline & & + psychosocial & 6 & 1 \\
\hline & $\begin{array}{l}\text { Immigrant worker/ } \\
\text { profesional }\end{array}$ & + psychosocial & 5 & 5 \\
\hline OECD & Immigrant & $\begin{array}{l}+ \text { worker }+ \\
\text { psychosocial }\end{array}$ & 9 & 0 \\
\hline \multirow[t]{3}{*}{ SCIELO } & \multirow[t]{3}{*}{ Inmigrante (93) } & + psicosocial & 1 & 0 \\
\hline & & + España & 53 & \\
\hline & & + España + mental & 5 & 5 \\
\hline \multirow[t]{4}{*}{ EMBASE } & \multirow[t]{2}{*}{ Immigrants (7042) } & + psychosocial disease & 33 & 8 \\
\hline & & + occupational disease & 61 & \\
\hline & \multirow{2}{*}{$\begin{array}{l}\text { Immigrant workers } \\
\text { (127) }\end{array}$} & + psychosocial disease & 32 & \\
\hline & & + occupational disease & 71 & \\
\hline
\end{tabular}




\begin{tabular}{|c|c|c|c|c|}
\hline & \multicolumn{2}{|c|}{$\begin{array}{c}\text { Términos incluidos en la estrategia } \\
\text { de búsqueda }\end{array}$} & \multirow{2}{*}{$\begin{array}{c}\begin{array}{c}\text { N. }{ }^{\circ} \text { de artículos } \\
\text { resultado } \\
\text { de la búsqueda }\end{array} \\
4\end{array}$} & \multirow{2}{*}{$\begin{array}{c}\begin{array}{c}\mathbf{N} .^{\circ} \text { de artículos } \\
\text { seleccionados } \\
\text { por resumen (abstract) }\end{array} \\
3\end{array}$} \\
\hline \multirow[t]{4}{*}{ IBECS } & \multirow[t]{2}{*}{ Immigrants (7042) } & + psychosocial disease & & \\
\hline & & + occupational disease & 1 & \\
\hline & \multirow{2}{*}{$\begin{array}{l}\text { Immigrant workers } \\
\text { (127) }\end{array}$} & + psychosocial & 3 & \\
\hline & & + occupational disease & 9 & \\
\hline \multirow[t]{5}{*}{ LILACS } & Immigrant (84) & + psychosocial disease & 3 & 1 \\
\hline & \multirow[t]{2}{*}{ Immigrants (184) } & + psychosocial disease & 2 & \\
\hline & & + occupational disease & 1 & \\
\hline & \multirow[t]{2}{*}{ Immigrant workers } & + psychosocial disease & 0 & \\
\hline & & + occupational disease & 3 & \\
\hline \multirow[t]{4}{*}{ PsychInfo } & Immigrants (10150) & + psychosocial disease & 12 & 3 \\
\hline & \multirow{3}{*}{$\begin{array}{l}\text { Immigrants } \\
\text { workers (512) }\end{array}$} & + psychosocial & 9 & \\
\hline & & + psychosocial disease & 2 & \\
\hline & & + occupational disease & 4 & \\
\hline $\begin{array}{l}\text { Artículos } \\
\text { relacionados }\end{array}$ & & & 1 & 1 \\
\hline $\begin{array}{l}\text { Total artículos } \\
\text { seleccionados }\end{array}$ & & & 752 & 137 \\
\hline
\end{tabular}

A partir de la colección bibliográfica inicialmente obtenida, se procedió a realizar una primera selección eliminando duplicados, aplicando criterios de pertinencia y los siguientes criterios de inclusión y exclusión:

Criterios de inclusión: Artículos realizados en humanos, publicados durante los últimos 10 años (2002-2012), escritos en inglés o español, que abordasen el tema de las condiciones laborales de los trabajadores inmigrantes en España y sus repercusiones en el ámbito psicosocial, familiar y sociocultural, con independencia de su país de origen, sexo y ocupación.

Criterios de exclusión: Artículos publicados con anterioridad a 2002, que trataban sobre inmigración nacional, sobre población menor de 16 años o mayor de 70, y aquellos que no abordasen la patología mental o socio-laboral. Se rechazaron también documentos que no fuesen artículos originales, tipo cartas al director, capítulos monográficos o revisiones sistemáticas, aunque estos fueron de utilidad para centrar el marco de referencia tratado y la comparación de resultados en el apartado de discusión.

Para clasificar los artículos por nivel de evidencia se tuvieron en cuenta los Criterios del Scottish Intercollegiate Guidelines Network (SIGN).

\section{RESULTADOS}

Inicialmente se obtuvo una colección bibliográfica de 752 artículos, que tras eliminar duplicados y seleccionar en base a criterios de pertinencia quedaron 137 artículos a los que se aplicaron los criterios de inclusión y exclusión, quedando finalmente un total de 15 artículos para el estudio como se muestra en la Tabla II. 


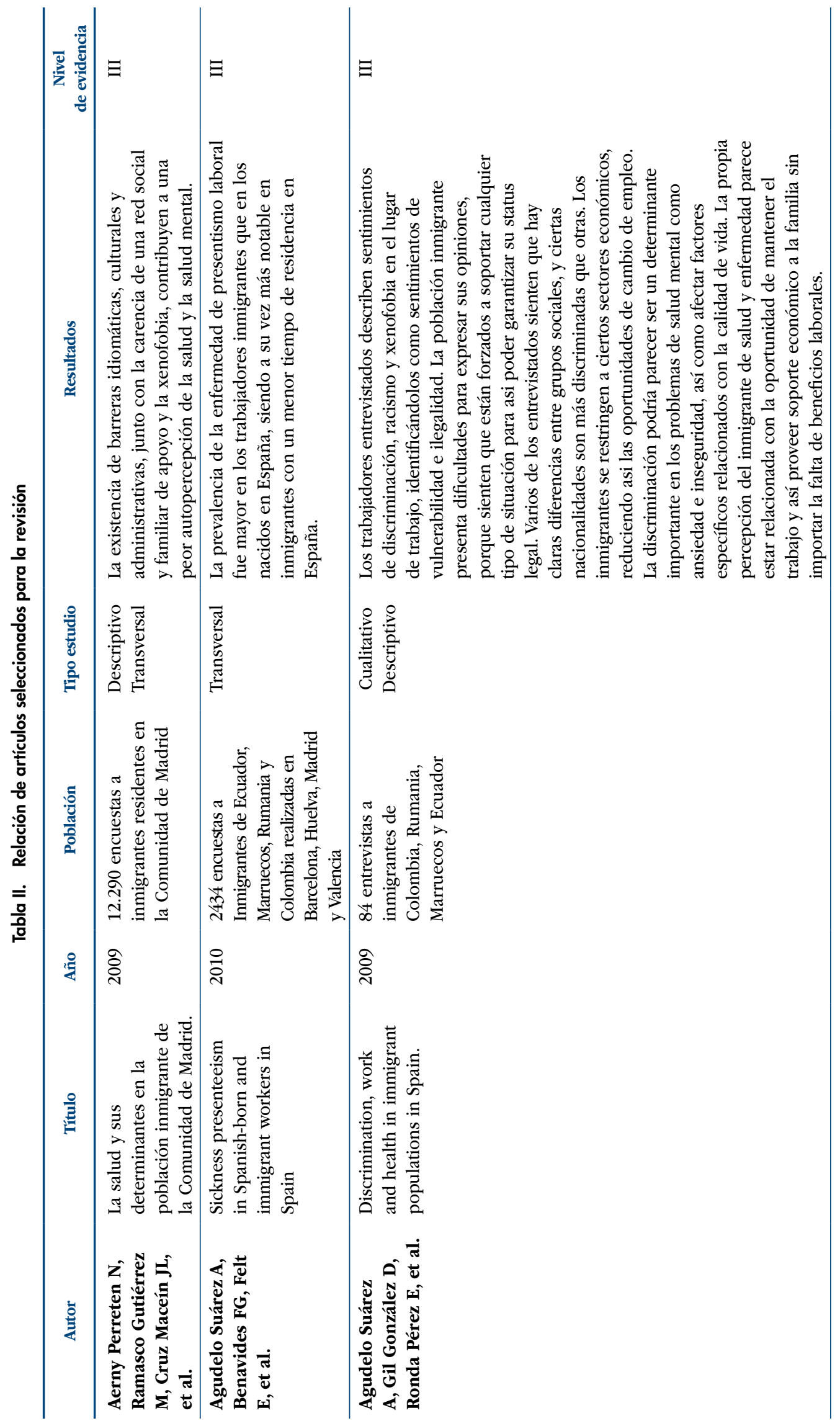




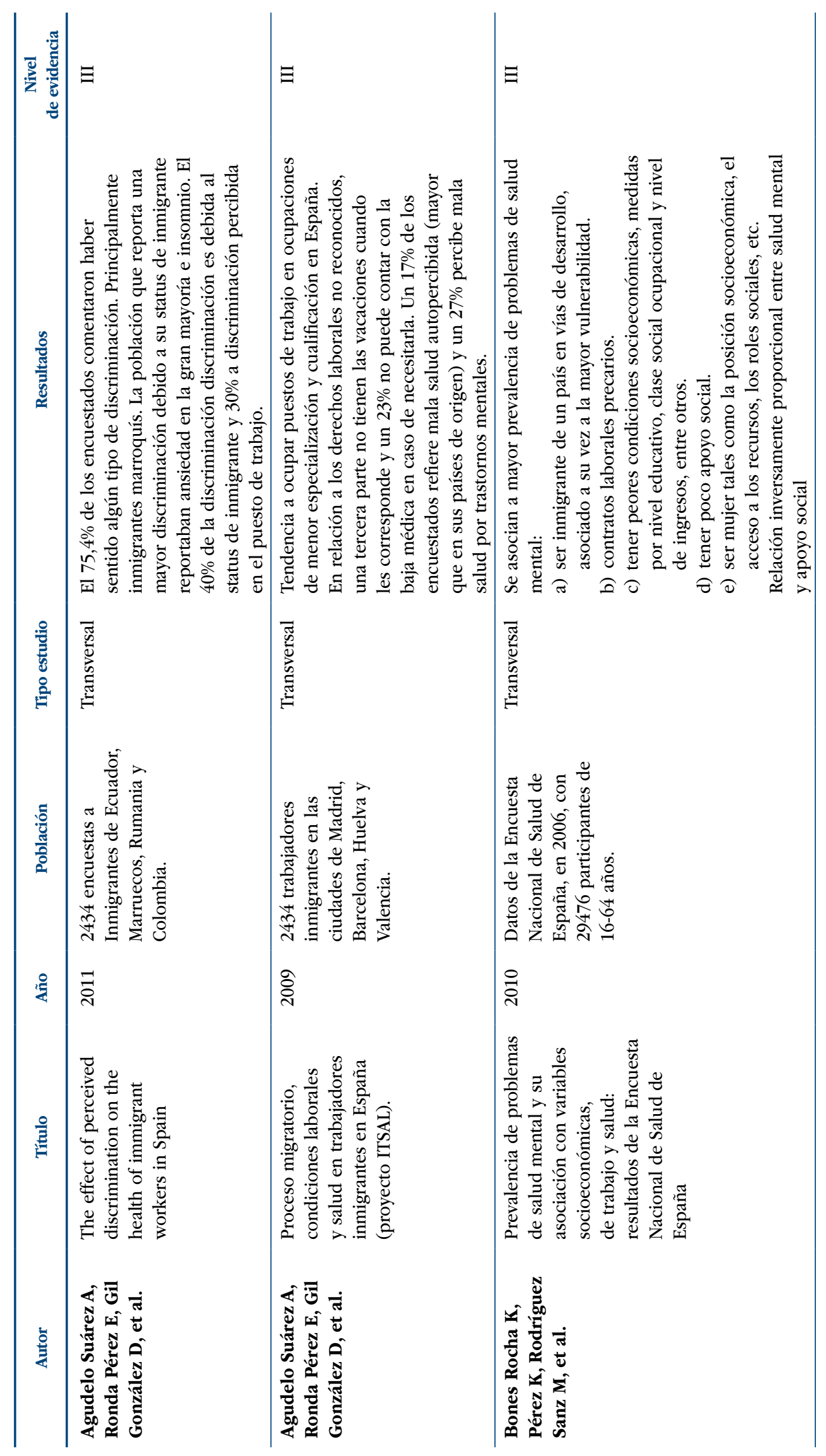




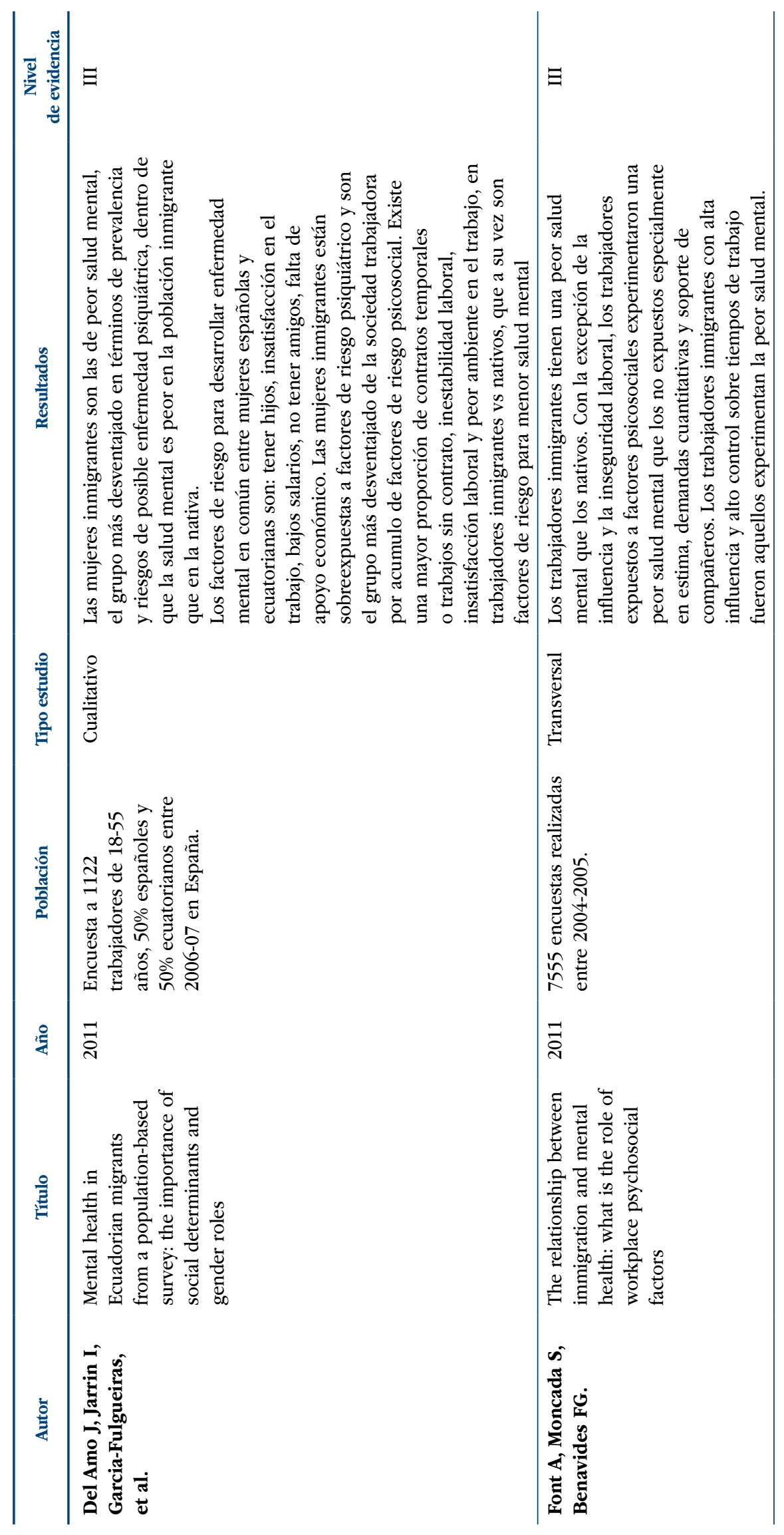




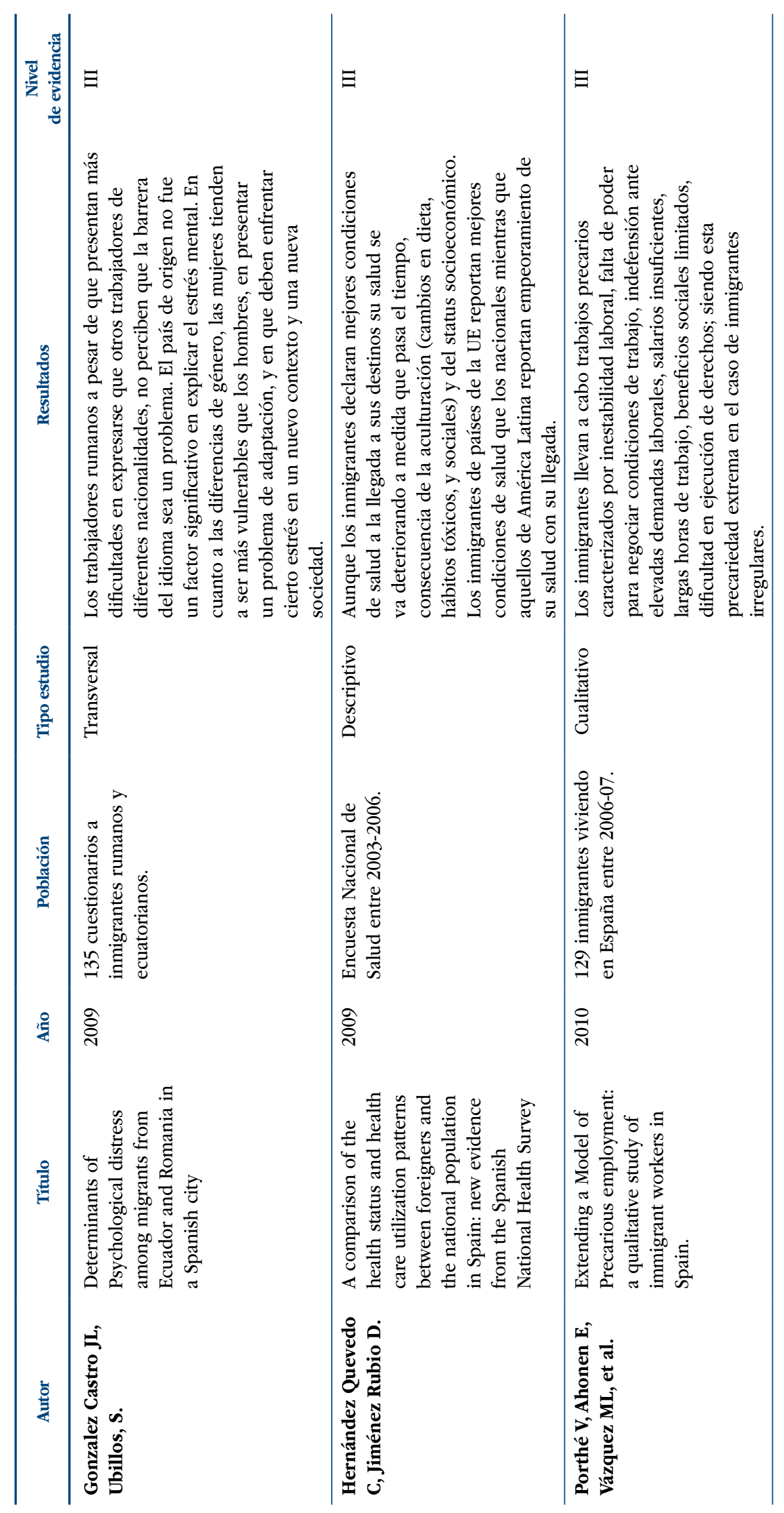




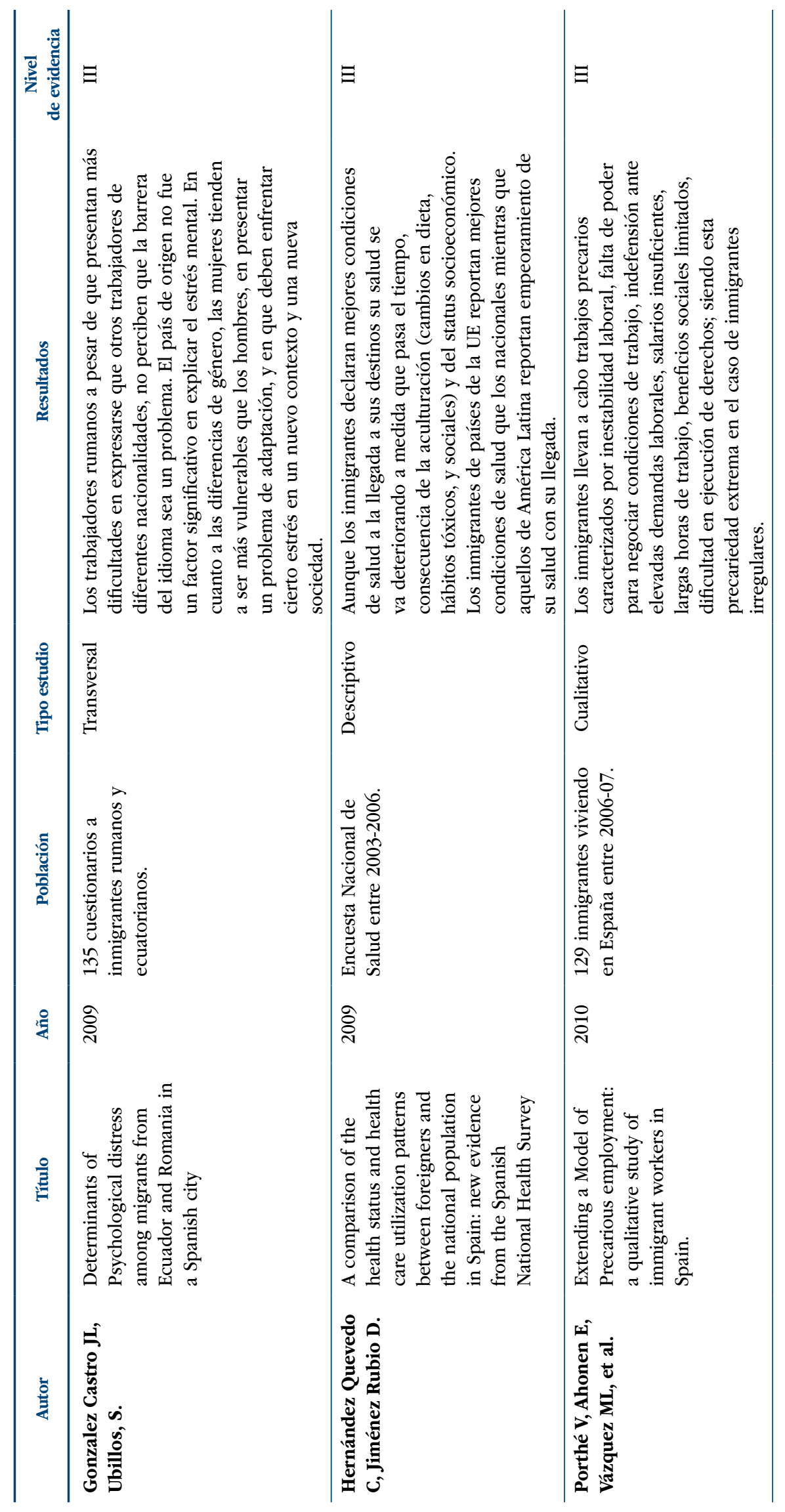




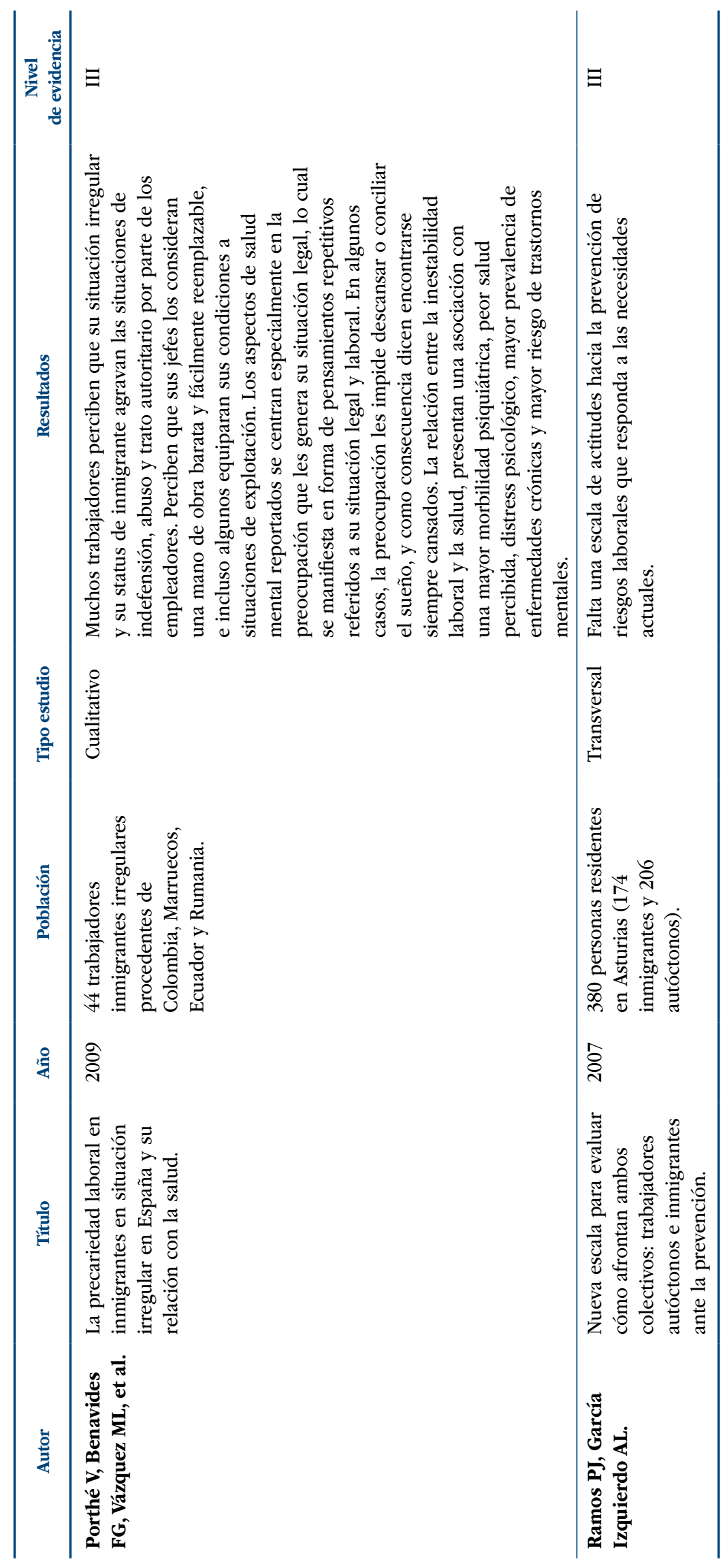


Durante el primer periodo de estudio (2002-2006) no se encuentra ninguna publicación, al igual que de forma aislada en 2008. La mayor parte de la producción científica se localiza en el año 2009 con un total de 7 artículos publicados (46\%), seguido de 2010 y 2011, ambos con 3 publicaciones (20\%) y sólo un artículo publicado (6\%) en los años 2007 y 2012. Esto indica que la preocupación dentro de la comunidad científica por este tema se despertó a partir del año 2009.

La mayor parte de los estudios se corresponden con estudios trasversales, 10 de los $15(66,6 \%)$ y $5(33,3 \%)$ son estudios cualitativos.

En lo que respecta a los principales hallazgos y resultados de los estudios, todos ellos encuentran una asociación entre determinadas condiciones psicosociales relacionadas con factores sociolaborales ligados a la migración y la mayor prevalencia de problemas de salud mental. La relación entre la inestabilidad laboral y la salud presenta una asociación con una mayor morbilidad psiquiátrica, peor salud percibida, distress psicológico, mayor prevalencia de enfermedades crónicas y mayor riesgo de trastornos mentales.

Los inmigrantes procedentes de países en vías de desarrollo presentan mayor vulnerabilidad, encontrándose frecuentemente ante situaciones de indefensión, abuso y trato autoritario por parte de los empleadores. En su gran mayoría reportan ansiedad acompañada de insomnio y algunos también describen sentimientos de discriminación, racismo y xenofobia en el lugar de trabajo, que aunque era percibido como un comportamiento dentro del marco de la ilegalidad, les producía una sensación de vulnerabilidad.

Muchos de los trabajadores inmigrantes desarrollan su trabajo mediante contratos laborales precarios y tienen peores condiciones socioeconómicas que el resto de la población en igualdad de condiciones, medidas mediante indicadores de nivel educativo, clase social ocupacional y nivel de ingresos, entre otros.

La existencia de barreras idiomáticas, culturales y administrativas, junto con la carencia de una red social, apoyo familiar y situaciones de xenofobia, contribuyen a una peor autopercepción de la salud tanto física como mental.

Las mujeres tienden a ser más vulnerables que los hombres, a presentar mayores problemas de adaptación, pues deben enfrentar cierto estrés en un nuevo contexto y una nueva sociedad. Las inmigrantes parecen presentar peor salud mental, considerándose el grupo más desventajado en términos de prevalencia y riesgos de posible enfermedad psiquiátrica, partiendo de la base de que la salud mental es peor ya de por si en la población inmigrante que en la nativa.

La mayor parte de los inmigrantes en España provienen de países menos desarrollados $y$, a pesar de tener estudios, es frecuente que se dediquen a desarrollar trabajos poco cualificados, lo que puede ser la causa de que se detecte mayor proporción de trabajadores inmigrantes expuestos a riesgos psicosociales que entre el resto de la población.

Por último, las características laborales en las que se encuentra una gran parte de las poblaciones inmigrante, les obliga a restringir a su entorno social dentro de determinados sectores socioeconómicos, reduciendo así las oportunidades de cambio o mejora de empleo.

\section{DISCUSIÓN}

La inmigración en España es un tema de actualidad, especialmente en la última década, en la que ha existido un rápido crecimiento de la inmigración en nuestro país, lo que plantea la necesidad de pensar los procedimientos estructurales de integración social que den cohesión a una sociedad multicultural. Sin embargo, el interés por su estudio desde el punto de vista psicosocial parece tomar impulso a partir del 2009. 
La mayor parte de los autores coinciden en que las condiciones de trabajo del inmigrante en España están caracterizadas por la precariedad, inestabilidad y consecuentemente vulnerabilidad en el ámbito laboral, especialmente en inmigrantes en situación irregular ${ }^{4,8-12}$

En comparación con el trabajador nativo, la población inmigrante desarrolla más ocupaciones que no se corresponden con su nivel educativo, en especial el género femenino ${ }^{8}$, accediendo a puestos no cualificados, con alta inestabilidad, peores condiciones generales y sueldos más bajos, lo que genera en ellos una "incertidumbre vital" que está relacionada directamente con mayor nivel de estrés y ansiedad, propiciando la aparición de un mayor número de trastornos mentales menores además de manifestaciones psicosomáticas ${ }^{4}$. Estas conclusiones ya fueron observadas en estudios previos publicados anteriormente al periodo de nuestro estudio, y en los que se destaca que la informalidad en el empleo puede asociarse a una mayor morbilidad psiquiátrica, peor salud autopercibida, distrés psicológico, mayor prevalencia de enfermedades crónicas y mayor riesgo de trastornos mentales ${ }^{13-15}$

Otro de los factores analizados en el entorno laboral es la discriminación percibida en relación a la población nativa en cuanto a remuneración, tareas asignadas, y tipo de trabajo y horarios ${ }^{4,16,17}$, así como la dificultad para acceder a algunos tipos de trabajos (por ejemplo el servicio civil) y la necesidad de soportar cualquier tipo de situación para poder mantener su trabajo y su situación de legalidad. Estos hechos contribuyen directamente a una situación de ansiedad, preocupación e inseguridad que se consideran determinantes en la aparición de problemas de salud mental ${ }^{16}$. En cifras, según el estudio de Agudelo-Suárez et al. ${ }^{17}$, la gran mayoría de inmigrantes encuestados $(75,4 \%)$ comentaron haber sentido algún tipo de discriminación, principalmente la población marroquí; hasta el $40 \%$ percibieron discriminación por su estatus de inmigrante y el $30 \%$ por su puesto de trabajo. Estas cifras nos hacen reflexionar sobre la importancia de identificar la raíz de las causas de la desigualdad, y además, nos lleva a plantearnos si España ofrece un marco receptivo y abierto a la población extranjera o siguen permaneciendo bolsas resistentes a compartir y aceptar la diversificación de las culturas, aún en pleno siglo XXI, cuya principal característica es la multiculturalidad y la globalización.

Otros determinantes de la salud mental de los inmigrantes fueron los factores psicosociales dentro del entorno laboral, demostrándose que aquellos inmigrantes que estuvieron sometidos a alta demanda cuantitativa y emocional, bajas posibilidades para el desarrollo, escaso apoyo y baja estima por parte de los compañeros, percibieron peor salud mental que el resto de los trabajadores ${ }^{18,19}$.

Font et al. demuestran que la población inmigrante tiene mayor probabilidad de estar en situación desfavorable para cada uno de los riesgos psicosociales (estima, control sobre horarios y apoyo social de compañeros de trabajo, entre otros), siendo los inmigrantes con trabajos manuales y las mujeres inmigrantes aquellos con más alta prevalencia de factores psicosociales desfavorables ${ }^{19}$.

En un marco general podemos considerar que los datos comentados presentan un patrón similar a los encontrados en previas revisiones sistemáticas, confirmándose que el estatus del inmigrante, es decir, la posición que ocupa dentro de la sociedad, se constituye en un eje de desigualdad social que, en el caso de los trabajadores, se combina con su situación de irregularidad y con una precariedad económica que además puede tener consecuencias negativas sobre la salud mental y física ${ }^{20,21}$.

Varios artículos hacen referencia a las diferencias de género, país de procedencia y tipo de trabajo en los riesgos psicosociales.

Respecto a las diferencias según el sexo, González y Ubillos, destacan en su estudio que las mujeres son más vulnerables para presentar problemas de adaptación, aunque tienen también más capacidad para buscar ayuda ${ }^{22}$. Aerny-Perreten, hace mención especial al alto porcentaje de mujeres inmigrantes que desarrollan ocupaciones que no se corresponden con su nivel educativo $(54,6 \%$ de inmigrantes universitarias tienen una 
ocupación manual, respecto al 6,2\% nacidas en España con el mismo nivel educativo) ${ }^{8}$. Kirchner y Patiño concluyen que las mujeres inmigrantes, comparadas con las nativas, reportan niveles más elevados de clínica psicológica ${ }^{23}$. Y, Del Amo et al., destacan que las mujeres inmigrantes son el grupo más desventajado en términos de prevalencia y riesgos de enfermedades mentales ${ }^{24}$

Los hallazgos encontrados parecen apuntar a que la mujer en este terreno es desfavorecida. Y, analizando las causas de estos hechos, nos planteamos la relación con el tipo de trabajo desempeñado más frecuentemente por las mujeres inmigrantes, como puede ser el trabajo doméstico o el cuidado de ancianos y niños, ambos realizados en soledad y que presentan mayor inestabilidad de horarios con bajos salarios, a lo que se les une el menor potencial para el desarrollo de capacidades personales. Además, en muchas ocasiones la propia migración supone un cambio de rol social y de género en relación al que tenía en su país ${ }^{24}$.

Pocos estudios hacen mención a las diferencias encontradas según países de procedencia geográfica. González Castro y Ubillos concluyen en su estudio, que el país de origen y la barrera idiomática no fueron factores significativos en justificar el estrés mental ${ }^{22}$. Hernández-Quevedo y Jiménez-Rubio afirman que los inmigrantes procedentes de la Unión Europea reportan mejores condiciones de salud que los nacionales, mientras que aquellos de América Latina aquejan empeoramiento de su salud con su llegada a nuestro país ${ }^{24,25}$.

Respecto al tipo de trabajo desarrollado, únicamente se encontró un artículo que destacase que aquellos inmigrantes con trabajos manuales tienen mayor prevalencia de riesgo psicosocial que otras poblaciones trabajadoras. Quizá estudios posteriores abordando este tema puedan arrojar más luz sobre el asunto ${ }^{19}$.

Si uno de los planteamientos al inicio de la revisión era conocer cuáles serían las características de la población inmigrante en España, Agudelo-Suárez et al. nos aportan datos a partir de los resultados del proyecto ITSAL (2009), en el que encontramos que el $90 \%$ de encuestados es menor de 45 años, el 51\% tienen estudios de formación profesional y bachillerato, un $70 \%$ afirma tener personas dependientes económicamente en sus países de origen, el 52\% haber emigrado por razones económicas y el $36 \%$ por razones laborales, el $76 \%$ tienen permiso de residencia y trabajo, el $72 \%$ tiene contrato de tipo temporal o está sin contrato, el 71\% está afiliado a la Seguridad Social, un 40\% trabaja más de 41 horas a la semana, una tercera parte no tiene las vacaciones cuando les corresponde y un $23 \%$ no puede contar con la baja médica en caso de necesitarla. Con respecto a la salud, el $17 \%$ de los inmigrantes encuestados refiere mala salud autopercibida (mayor que en sus países de origen) y un $27 \%$ percibe mala salud por trastornos mentales ${ }^{11}$.

Los especialistas en salud laboral se encuentran de acuerdo en que la migración es sólo un factor de riesgo psicosocial cuando se acompaña de situaciones sobreañadidas como la propia vulnerabilidad de la persona y/o un medio de acogida hostil ${ }^{26}$. Así pues, para comprender la relación que existe entre el proceso de migración, la exposición a sus factores de riesgo y el desarrollo de un problema de salud mental, es necesario tener presente una diversidad de dimensiones que componen el marco sociolaboral de los inmigrantes y que incluyen características de la persona, condiciones previas a la migración, características propias del proceso de migración y condiciones en la nueva sociedad donde se llega a residir ${ }^{27}$.

El síndrome del inmigrante con estrés crónico y múltiple o "Síndrome de Ulises" (haciendo mención al héroe griego que padeció innumerables adversidades y peligros lejos de sus seres queridos) se define como el cuadro reactivo de estrés que surge ante situaciones de duelo migratorio extremo (en el que se superan las capacidades de adaptación del sujeto), con clínica variable de esfera depresiva, ansiedad, somatización y confusional. Su presencia continua en la vida cotidiana puede terminar desencadenando patologías propiamente mentales, por lo que conviene realizar una adecuada prevención sanitaria y psicosocial y un diagnóstico precoz en su defecto para un temprano y 
multidisciplinar abordaje por parte de psicólogos, trabajadores sociales, enfermería, educadores sociales, entre otros $26,28,29$.

En cuanto a las medidas de prevención, no se han encontrado muchos estudios que las analicen en profundidad, precisando de mayor conocimiento científico acerca de este problema para buscar estrategias concretas de acción ${ }^{11}$. Ramos et al. proponen una escala de actitudes hacia la prevención de riesgos laborales que responda a las necesidades actuales en el trabajo, tanto para trabajadores inmigrantes como autóctonos, y valorar intervenciones específicas en el puesto de trabajo ${ }^{30}$. Mientras, Alvarado propone la necesidad de un entrenamiento especial para los trabajadores sanitarios en relación a la atención de los problemas de salud en población de inmigrantes y en especial en el campo de la salud mental ${ }^{27}$.

En todos los casos, se habla de intervenciones a nivel multidisciplinar y siempre haciendo presente la importancia del papel de la política social. Es fundamental el desarrollo de sistemas de evaluación epidemiológica, así como de políticas sociales y económicas decididas, que promuevan la solidaridad, la reducción de desigualdades, el respeto a los derechos laborales y la no discriminación por ser inmigrantes desde un enfoque de equidad. Requiriendo, en todos los casos, de un mayor conocimiento científico multidisciplinario acerca del problema, así como de una estrategia de acción coordinada entre diferentes niveles de gobierno y de carácter intersectorial.

\section{AGRADECIMIENTOS}

Los autores agradecen su inestimable colaboración a Jorge Veiga de Cabo Subdirector de la Escuela Nacional de Medicina del Trabajo.

\section{REFERENCIAS BIBLIOGRÁFICAS}

1. http://extranjeros.empleo.gob.es/es/Estadisticas/operaciones/con-certificado/201209/Principales _ resultados_30092012.pdf

2. http://www.oecd.org/migration/internationalmigrationpoliciesanddata/IMO\%202012_country\%20 note\%20SPAIN_ES_linked.pdf

3. Martínez-Moneo M y Martínez Larrea A, 2006. Patología psiquiátrica en el inmigrante. An. Sist. Sanit. Navar., 29, Supl. 1

4. Galvez Herrer M, Gutierrez García MD y Zapico Martínez N. 2011. Salud laboral y salud mental en población trabajadora inmigrante. Medicina y Seguridad del Trabajo, 57. (Supl. 1): 1-262.

5. Gil-Monte PR. 2009. Algunas razones para considerar los riesgos psicosociales en el trabajo y sus consecuencias en la salud pública. Revista Española de Salud Pública, 83, n. ${ }^{\circ} 2$, ISSN 1135-5727.

6. Ramasco M, Palanca I. Sistema sanitario ante la diversidad sociocultural en la ciudad de Madrid. Anuario de la convivencia intercultural. Ciudad de Madrid. Observatorio de las Migraciones y de la Convivencia Intercultural de la Ciudad de Madrid. Área de Gobierno de Empleo y Servicios a la Ciudadanía del Ayuntamiento de Madrid; 2006; 377-85.

7. Berra S, Eloraza Ricart JM, Bartomeu N, et al. Necesidades en salud y utilización de los servicios sanitarios en la población inmigrante en Cataluña. Revisión exhaustiva de la literatura científica. Barcelona: Agencia d'Avaluacio de Tecnologia i Recerca Mediques; 2004.

8. Aerny-Perreten N, Ramasco Gutiérrez M, Cruz Maceín JL, Rodríguez Rieiro C, Garabato González S Rodríguez Laso A. 2010. La salud y sus determinantes en la población inmigrante de la Comunidad de Madrid. Gaceta Sanitaria, 24 (2): 136-144.

9. García et al. 2009. Condiciones de trabajo y salud en inmigrantes (Proyecto ITSAL): entrevistas a informantes clave. Gaceta Sanitaria; 2009; 23 (2): 91-99.

10. Porthé V, Benavides FG, Vázquez ML, Ruiz-Frutos C, García AM, Ahonen E, Agudelo-Suárez AA, Benach J. Proyecto ITSAL. La precariedad laboral en inmigrantes en situación irregular en España y su relación con la salud. Gaceta Sanitaria; 2009; 1: 107-14.

11. Agudelo-Suárez. 2009. Proceso migratorio, condiciones laborales y salud en trabajadores inmigrantes en España (proyecto ITSAL). Gaceta Sanitaria, 23, Suppl. 1: 115-21. 
12. Porthé V, Ahonen E, Vázquez ML, Pope C, Agudelo AA, García AM, Amable M, Benavides FG, Benach J. ITSAL Project. 2010. Extending a model of precarious employment: A qualitative study of immigrant workers in Spain. American Journal Industrial Medicine, 53: 417-24.

13. Ferrie JE. 2001. Is job insecurity harmful to health? J R Soc Med, 94: 71-76.

14. Virtanen P, Vahtera J, Kivimäki M, et al. 2002. Employement security and health. Journal of Epidemiology Community Health, 56: 569-74

15. Ludermir AB, Lewis G. 2003. Informal work and common mental disorders. Soc psychiatry Psychiatr Epidemiol, 38: 485-9.

16. Agudelo-Suárez A, Gil González D, Ronda Pérez E, Porthé V, Paramio-Pérez G, García AM, Garí A. 2009. Discrimination, work and health in immigrant population in Spain. Soc Sci Med., 68: 1866-74.

17. Agudelo-Suárez AA, Ronda Pérez E, Gil González D, Vives Cases C, García AM, Ruiz-Frutos C, Felt E, Benavides FG. 2011. The effect of perceived discrimination on the health of immigrant workers in Spain. BMC Public Health, 11: 652.

18. Font A, Moncada S y Benavides FG. 2012. The relationship between immigration and mental health: what is the role of workplace psychosocial factors. International Archives of Occupational and Environmental Health, 85: 801-806. doi: 10.1007/s00420-011-0724-6.

19. Font A, Moncada S, Llorens C y Benavides FG. 2012. Psychosocial factor exposures in the workplace: differences between immigrants and Spaniards. European Journal of Public Health, 22: 688-93.

20. Benach J, Muntaner C, Chung H, Benavides FG. 2010. Immigration, Employment relations, and Health: developing a research agenda. American Journal of Industrial Medicine, 53 (4): 338-43.

21. Porthé V, Amable M, Benach J. 2007. La precariedad laboral y la salud de los inmigrantes en España: ¿qué sabemos y qué deberíamos saber? Archivos Prevención Riesgos Laborales, 10 (1): 34-39.

22. Gonzalez-Castro JL y Ubillos, S. 2009. Determinants of Psychological distress among migrants from Ecuador and Romania in a Spanish city. International Journal of Social Psychiatry, 57 (1): 30-44.

23. Kirchner T y Patiño C. 2011. Latin-American immigrant women and mental health: differences according to their rural or urban origin. Spanish Journal of Psychology, 14 (2): 843-50.

24. Del Amo J, Jarrin I, Garcia-Fulgueiras, et al. 2011. Mental health in Ecuadorian migrants from a populationbased survey: the importance of social determinants and gender roles. Social Psychiatry \& Psychiatric Epidemiology, 46 (11): 1143-1152.

25. Hernández-Quevedo C y Jiménez-Rubio D. 2009. A comparison of the health status and health care utilization patterns between foreigners and the national population in Spain: new evidence from the Spanish National Health Survey. Soc Sci Med., 69 (3): 370-8.

26. Achotegui J. 2008. Migración y crisis: El síndrome del inmigrante con estrés crónico y múltiple (síndrome de Ulises). Avances en Salud Mental Relacional, 7 (1).

27. Alvarado, R. 2008. Salud mental en inmigrantes. Revista Chilena de Salud Pública, 12: 37-41.

28. Achotegui J. 2009. Migración y salud mental. El síndrome del inmigrante con estrés crónico y múltiple (síndrome de Ulises). Abendua, Zerbitzuam, 46.

29. Achotegui J. 2008. Duelo migratorio extremo: el síndrome del inmigrante con estrés crónico y múltiple (síndrome de Ulises). Revista de Psicopatología y salud mental del niño y del adolescente, ISSN 16958691, n. $^{\circ} 11,15-25$.

30. Ramos Villagrasa PJ, León García-Izquierdo A. 2007. Nueva escala para evaluar cómo afrontan ambos colectivos: trabajadores autóctonos e inmigrantes ante la prevención. Mapfre Seguridad, 107.

||||||||||||||||||||||||||||||||||||||||||||||||||||||||||||||||||||||||||||||||||||||||||||||||||||||||||||||||||||||||||||||||||||||||||||||||||||||||||||||||||||||||||||||||||||||||||||||||||||||||| || 